\title{
RLS Wiener Smoother for Colored Observation Noise with Relation to Innovation Theory in Linear Discrete-Time Stochastic Systems
}

\author{
Seiichi Nakamori \\ Department of Technical Education, Kagoshima University, Kagoshima 890-0065, Japan \\ nakamori@edu.kagoshima-u.ac.jp
}

\begin{abstract}
Almost estimators are designed for the white observation noise. In the estimation problems, rather than the white observation noise, there might be actual cases where the observation noise is colored. This paper, from the viewpoint of the innovation theory, based on the recursive least-squares (RLS) Wiener fixed-point smoother and filter for the colored observation noise, newly proposes the RLS Wiener fixed-interval s moothing algorithm in linear discretetime wide-sense stationary stochastic systems. The observation $y(k)$ is given as the sum of the signal $z(k)=H x(k)$ and the colored observation noise $v_{c}(k)$. The RLS Wiener fixed-interval smoother uses the following information: (a) the system matrix for the state vector $x(k)$; (b) the observation matrix $H$; (c) the variance of the state vector; (d) the system matrix for the colored observation noise $v_{c}(k)$; (e) the variance of the colored observation noise; (f) the input noise variance in the state equation for the colored observation noise.
\end{abstract}

Index Terms - Discrete-Time Stochastic System, RLS Wiener Fixed-Interval Smoother, Colored Observation Noise, Covariance Information, Innovation Theory

\section{Introduction}

In comparis on with the Kalman estimators, the RLS Wiener estimators are advantageous in the point that the RLS Wiener estimators do not use the information of the input noise variance and the input matrix in the state equation for the signal. The less information in the estimators might avoid the degradation of the estimation accuracy caused by the inaccurate information on the state-space model. In [1], the RLS W iener filter and fixed-point smoother are proposed in linear discretetime stochastic systems. The estimators need the information of the system matrix, the observation vector, the variance of the state vector and the variance of white observation noise. In addition, in linear discretetime stochastic systems, the following RLS Wiener estimators are studied, i.e. the Chandrasekhar-type RLS Wiener fixed-point s moother, filter and predictor [2], the square-root RLS Wiener fixed-point s moother and filter [3] and the RLS Wiener FIR filter [4], etc.

Almost estimators are designed for the white observation noise. In the estimation problems, there might be actual cases where the observation noise is colored. The estimation problem for the observation equation, with additive colored observation noise, has received much attention in the detection and estimation problems for communication systems. For example, in [5]-[10], the estimation problem is considered in linear discrete-time stochastic systems. In [8], an alternative method is proposed on the traditional handling of the autoregressive colored observation noise in the speech enhancement algorithm based on the Kalman filter. In [10], based on the autoregressive moving average (ARMA) innovation model, the reduced-order Wiener estimators for descriptor system with MA colored observation noise and multi-observation lags are presented.

In [11], for the white observation noise, the signal estimation problem is considered in linear continuoustime stochastic systems. Also, the spectral factorization method is discussed on the system matrix, the input matrix and the observation matrix. That is, the innovation state-space model for the colored observation model is developed.

In [12], based on the improved least-squares (ILS) method, the parameter estimation technique for a noisy autoregressive (AR) signal, observed with additive colored noise, is presented.

In [13], starting with the Wiener-Hopf equation, the RLS Wiener fixed-point smoother and filter are presented for the colored observation noise. By the way, the innovation theory is an interesting method, which has been investigated in the area of the estimation techniques for the white observation noise. It might be worthwhile to consider the RLS Wiener smoothing problem also for the colored observation noise. This paper, with the relation of the work in [13] to the innovation theory [14], [15], newly proposes the discrete-time smoother for the colored observation noise in linear wide-sense stationary stochastic systems. It is assumed that the smoothing estimate is given as a linear transformation of the innovation process. The 
approach, adopted in this paper, to the design of the RLS Wiener s moother has not be examined hitherto in the case of the colored observation noise. Based on the RLS Wiener smoother, the fixed-interval s moothing algorith $\mathrm{m}$ for the colored observation noise is shown in the flowchart of Fig. 1. The observation $y(k)$ is given as a sum of the signal $z(k)=H x(k)$ and the colored observation noise $v_{c}(k)$. The RLS Wiener estimators use the following information: (a) the system matrix for the state vector $x(k)$; (b) the observation matrix $H$; (c) the variance of the state vector; (d) the system matrix for the colored observation noise $v_{c}(k)$; (e) the variance of the colored observation noise; (f) the input noise variance in the state equation for the colored observation noise. Also, the filtering error variance function $\tilde{P}_{F}(L, L)$ and the prediction error variance function $\tilde{P}_{P}(L, L-1)$, for the signal $z(L)$, are formulated with regard to the current RLS Wiener estimators.

In section 2, the smoothing problem, based on the innovation theory, is introduced. In section 3, Theorem 1 proposes the RLS Wiener smoothing and filtering algorithms. In section 4, Fig. 1 depicts the flowchart for the fixed-interval smoothing estimate $\hat{z}(k, L)$.

A numerical simu lation example, in section 4, shows the estimation characteristics of the proposed RLS Wiener fixed-interval smoother and filter for the colored observation noise.

\section{Least-Squares Smoothing Problem Based on Innovation Theory}

Let m-dimensional observation equation be described by

$$
y(k)=z(k)+v_{c}(k), z(k)=H x(k)
$$

in linear discrete-time stochastic systems. Here, $H$ is an $m \times n$ observation matrix, $z(k)$ is the zero-mean signal vector. The process $\left\{v_{c}(k), k \geq 0\right\}$ represents the zero-mean colored observation noise sequence. It is assumed that the signal is uncorrelated with the colored observation noise as

$$
E\left[z(k) v_{c}^{T}(s)\right]=0,0 \leq k, s<\infty .
$$

Let $K_{x}(k, s)=K_{x}(k-s)$ represent the auto-covariance function of the state vector $x(k)$ in wide-sense stationary stochastic systems [16], and let $K_{x}(k, s)$ be expressed in the form of

$$
\begin{aligned}
& K_{x}(k, s)= \begin{cases}A(k) B^{T}(s), & 0 \leq s \leq k, \\
B(s) A^{T}(k), & 0 \leq k \leq s,\end{cases} \\
& A(k)=\Phi^{k}, \quad B^{T}(s)=\Phi^{-s} K_{x}(s, s) .
\end{aligned}
$$

Here, $\Phi$ is the transition matrix of $x(k)$.

Let the state-space model for $x(k)$ be described as

$$
\begin{aligned}
& x(k+1)=\Phi x(k)+G w(k), \\
& E\left[w(k) w^{T}(s)\right]=Q(k) \delta_{K}(k-s),
\end{aligned}
$$

where $G$ is an $n \times l$ input matrix and $w(k)$ is the white input noise vector with the auto-covariance function of (4).

Let $K_{c}(\cdot, \cdot)$ denote the auto-covariance function of $v_{c}(k)$. The auto-covariance function $K_{c}(k, s)$ is given by

$$
\begin{aligned}
& K_{c}(k, s)= \begin{cases}A_{c}(k) B_{c}^{T}(s), & 0 \leq s \leq k, \\
B_{c}(s) A_{c}^{T}(t), & 0 \leq k \leq s,\end{cases} \\
& A_{c}(k)=\Phi_{c}^{k}, \quad B_{c}^{T}(s)=\Phi_{c}^{-s} K_{c}(s, s) .
\end{aligned}
$$

Let the state equation for $v_{c}(k)$ be given by

$$
\begin{aligned}
& v_{c}(k+1)=\Phi_{c} v_{c}(k)+u(k), \\
& E\left[u(k) u^{T}(s)\right]=R_{u}(k) \delta_{K}(k-s),
\end{aligned}
$$

in terms of the white input noise vector $u(k)$ with the variance $R_{u}$. It is found that for the expressions $K_{c}(k+1, k+1)=E\left[v_{c}(k+1) v_{c}^{T}(k+1)\right]$, $K_{c}(k, k)=E\left[v_{c}(k) v_{c}{ }^{T}(k)\right]$, in the wide-sense stationary stochastic systems, the following relationships hold.

$$
\begin{aligned}
& R_{u}(k)=K_{c}(k+1, k+1)-\Phi_{c} K_{c}(k, k) \Phi_{c}^{T}, \\
& K_{c}(k+1, k+1)=K_{c}(k, k)=K_{c}(0)
\end{aligned}
$$

It is shown that the fixed-interval s moothing estimate $\hat{x}(k, L)$ of $x(k)$ is given by

$$
\begin{aligned}
& \hat{x}(k, L)=\sum_{i=1}^{L} g(k, i) v(i)=\hat{x}(k, k)+\sum_{i=k+1}^{L} g(k, i) v(i), \\
& \hat{x}(k, k)=\sum_{i=1}^{k} g(k, i) v(i)
\end{aligned}
$$

in terms of the impulse response function $g(k, i)$ and the innovation process $\{v(i), \quad 1 \leq i \leq L\}$ [14], [15]. Also, $\hat{x}(k, k)$ represents the filtering estimate of $x(k)$, which is given as a linear transform of the innovation process $\{u(i), \quad 1 \leq i \leq k\}$.

Let us consider the estimation problem, which minimizes the mean-square value (MSV)

$$
J=E\left[\|x(k)-\hat{x}(k, L)\|^{2}\right]
$$

of the fixed-point smoothing error $x(k)-\hat{x}(k, L)$. From an orthogonal projection lemma [16], 


$$
x(k)-\sum_{i=1}^{L} g(k, i) v(i) \perp v(s), \quad 1 \leq s \leq L,
$$

the impulse response function satisfies the equation

$$
E\left[x(k) v^{T}(s)\right]=\sum_{i=1}^{L} g(k, i) E\left[v(i) v^{T}(s)\right] .
$$

Here ' $\perp$ ' denotes the notation of the orthogonality. From [13], the filtering estimate $\hat{x}(k, k)$ of $x(k)$ is calculated sequentially by

$$
\begin{aligned}
& \hat{x}(k, k)=\Phi \hat{x}(k-1, k-1)+G_{1}(k)(y(k) \\
& -\left(H \Phi \hat{x}(k-1, k-1)+\left(\Phi_{c}\right)^{2} \hat{v}_{2}(k-1, k-1)\right. \\
& \left.\left.+\Phi_{c} \hat{v}_{3}(k-1, k-1)\right)\right), \quad \hat{x}(0,0)=0 .
\end{aligned}
$$

It is clear that the innovation process $v(k)$ is expressed as

$$
\begin{aligned}
& v(k)=y(k)-(H \Phi \hat{x}(k-1, k-1) \\
& \left.+\Phi_{c}^{2} \hat{v}_{2}(k-1, k-1)+\Phi_{c} \hat{v}_{3}(k-1, k-1)\right) .
\end{aligned}
$$

Also, from (8) and (12), the filter gain $G_{1}(k)$ is equivalent to $g(k, k)$. For the innovation process satisfying

$$
E\left[v(k) v^{T}(s)\right]=\Delta(k) \delta_{K}(k-s),
$$

from (11), the impulse response function $g(k, s)$ and the filter gain $G_{1}(k)$ are given by

$$
g(k, s)=E\left[x(k) v^{T}(s)\right] \Delta^{-1}(s), G_{1}(k)=g(k, k) .
$$

From [13], it is seen that the variance $\Delta(L)$ of the innovation process $\{v(L), L \geq 0\}$ is formulated as (29) in Theorem 1.

\section{RLS Wiener Smoothing Equations}

Under the linear least-squares estimation problem of the signal $z(k)$ in section 2, Theorem 1 presents the RLS Wiener s moothing and filtering equations, which use the covariance information of the signal and the observation noise.

\section{Theorem 1}

Let the auto-covariance function $K_{x}(k, s)$ of the state vector $x(k)$ be expressed by (3), let the variance of the colored observation noise $v_{c}(k)$ be $K_{c}(k, k)$ and let the variance of $u(k)$ in the state equation (6) for $v_{c}(k+1)$ be $R_{u}(k)$. Then, the discrete-time RLS Wiener algorithms for the smoothing estimate and the filtering estimate of the signal $z(k)$ consist of (16)-(45) in linear wide-sense stationary stochastic systems.

Smoothing estimate of the signal $z(k): \hat{z}(k, L)$

$$
\hat{z}(k, L)=H \hat{x}(k, L)
$$

Smoothing estimate of $x(k): \hat{x}(k, L)$

$$
\begin{aligned}
& \hat{x}(k, L)=\hat{x}(k, k)+f_{0}(k+1, L)-f_{1}(k+1, L) \\
& \left.-f_{2}(k+1, L)-f_{3}(k+1, L)\right) \\
& f_{0}(k+1, L)=f_{0}(k+1, L-1) \\
& +K_{x}(k, k)\left(\Phi^{T}\right)^{L-k} H^{T} \Delta^{-1}(L) v(L), \\
& f_{0}(k+1, k+1)=K_{x}(k, k) \Phi^{T} H^{T} \Delta^{-1}(k+1) v(k+1) \\
& f_{1}(k+1, L)=f_{1}(k+1, L-1)+\left(d_{1}^{T}(k, L-1)\right. \\
& \left.+d_{2}^{T}(k+1, L-1)\right) H^{T} \Delta^{-1}(L) v(L), \\
& f_{1}(k+1, k+1)=S_{11}^{T}(k) \Phi^{T} H^{T} \Delta^{-1}(k+1) v(k+1) \\
& f_{2}(k+1, L)=f_{2}(k+1, L-1)+\left(d_{3}^{T}(k, L-1)\right. \\
& \left.+d_{4}^{T}(k+1, L-1)\right) \Delta^{-1}(L) v(L), \\
& f_{2}(k+1, k+1)=S_{21}^{T}(k)\left(\Phi_{c}^{T}\right)^{2} \Delta^{-1}(k+1) v(k+1) \\
& f_{3}(k+1, L)=f_{3}(k+1, L-1)+\left(d_{5}^{T}(k, L-1)\right. \\
& \left.+d_{6}^{T}(k+1, L-1)\right) \Delta^{-1}(L) v(L) \text {, } \\
& f_{3}(k+1, k+1)=S_{31}^{T}(k) \Phi_{c}^{T} \Delta^{-1}(k+1) v(k+1) \\
& d_{1}(k, L)=\Phi d_{1}(k, L-1)-\Phi G_{1}(L)\left(H d_{1}(k, L-1)\right. \\
& \left.+d_{3}(k, L-1)+d_{5}(k, L-1)\right) \text {, } \\
& d_{1}(k, k)=\Phi S_{11}(k) \\
& d_{2}(k+1, L)=\Phi d_{2}(k+1, L-1) \\
& +\Phi G_{1}(L)\left(H \Phi^{L-k} K_{x}(k, k)-H d_{2}(k+1, L-1)\right. \\
& \left.-d_{4}(k+1, L-1)-d_{6}(k+1, L-1)\right) \text {, } \\
& d_{2}(k+1, k+1)=\Phi G_{1}(k+1) H \Phi K_{x}(k, k) \\
& d_{3}(k, L)=\Phi_{c} d_{3}(k, L-1) \\
& -\Phi_{c}^{2} G_{2}(L)\left(H d_{1}(k, L-1)+d_{3}(k, L-1)\right. \\
& \left.+d_{5}(k, L-1)\right) \text {, } \\
& d_{3}(k, k)=\Phi_{c}^{2} S_{21}(k) \\
& d_{4}(k+1, L)=\Phi_{c} d_{4}(k+1, L-1) \\
& +\Phi_{c}^{2} G_{2}(L)\left(H \Phi^{L-k} K_{x}(k, k)\right. \\
& -H d_{2}(k+1, L-1)-d_{4}(k+1, L-1) \\
& \left.-d_{6}(k+1, L-1)\right) \text {, } \\
& d_{4}(k+1, k+1)=\Phi_{c}^{2} G_{2}(k+1) H \Phi K_{x}(k, k) \\
& d_{5}(k, L)=\Phi_{c} d_{5}(k, L-1) \\
& -\Phi_{c} G_{3}(L)\left(H d_{1}(k, L-1)+d_{3}(k, L-1)\right. \\
& \left.+d_{5}(k, L-1)\right) \text {, } \\
& d_{5}(k, k)=\Phi_{c} S_{31}(k) \\
& d_{6}(k+1, L)=\Phi_{c} d_{6}(k+1, L-1) \\
& +\Phi_{c} G_{3}(L)\left(H \Phi^{L-k} K_{x}(k, k)\right. \\
& -H d_{2}(k+1, L-1)-d_{4}(k+1, L-1) \\
& \left.-d_{6}(k+1, L-1)\right) \text {, } \\
& d_{6}(k+1, k+1)=\Phi_{c} G_{3}(k+1) H \Phi K_{x}(k, k)
\end{aligned}
$$


Innovation process: $v(L)$

$$
\begin{aligned}
& v(L)=y(L)-(H \Phi \hat{x}(L-1, L-1) \\
& \left.+\Phi_{c}^{2} \hat{v}_{2}(L-1, L-1)+\Phi_{c} \hat{v}_{3}(L-1, L-1)\right) .
\end{aligned}
$$

Variance of the innovation process $v(L): \Delta(L)$

$$
\begin{aligned}
& \Delta(L)=R_{u}(L)+\left(H K_{x}(L, L)-\left(H \Phi S_{11}(L-1) \Phi^{T}\right.\right. \\
& \left.\left.+\left(\Phi_{c}\right)^{2} S_{21}(L-1) \Phi^{T}+\Phi_{c} S_{31}(L-1) \Phi^{T}\right)\right) H^{T} \\
& +\left(\Phi_{c} K_{c}(L, L)-\left(H \Phi S_{12}(L-1) \Phi_{c}^{T}\right.\right. \\
& \left.\left.+\left(\Phi_{c}\right)^{2} S_{22}(L-1) \Phi_{c}^{T}+\Phi_{c} S_{32}(L-1) \Phi_{c}^{T}\right)\right) \Phi_{c}^{T} \\
& +\left(R_{u}(L)-\left(H \Phi S_{13}(L-1) \Phi_{c}^{T}\right.\right. \\
& \left.\left.+\left(\Phi_{c}\right)^{2} S_{23}(L-1) \Phi_{c}^{T}+\Phi_{c} S_{33}(L-1) \Phi_{c}^{T}\right)\right) .
\end{aligned}
$$

Filtering estimate of the signal $z(L): \hat{z}(L, L)$

$$
\hat{z}(L, L)=H \hat{x}(L, L)
$$

Filtering estimate of $x(L): \hat{x}(L, L)$

$$
\begin{aligned}
& \hat{x}(L, L)=\Phi \hat{x}(L-1, L-1)+G_{1}(L)(y(L) \\
& -\left(H \Phi \hat{x}(L-1, L-1)+\left(\Phi_{c}\right)^{2} \hat{v}_{2}(L-1, L-1)\right. \\
& \left.\left.+\Phi_{c} \hat{v}_{3}(L-1, L-1)\right)\right), \quad \hat{x}(0,0)=0
\end{aligned}
$$

Filtering estimate of $v_{c}(L)$ :

$$
\begin{aligned}
& \left.\Phi_{c} \hat{v}_{2}(L, L)+\hat{v}_{3}(L, L)\right) \\
& \hat{v}_{2}(L, L)=\Phi_{c} \hat{v}_{2}(L-1, L-1)+G_{2}(L)(y(L) \\
& -\left(H \Phi \hat{x}(L-1, L-1)+\left(\Phi_{c}\right)^{2} \hat{v}_{2}(L-1, L-1)\right. \\
& \left.\left.+\Phi_{c} \hat{v}_{3}(L-1, L-1)\right)\right), \quad \hat{v}_{2}(0,0)=0 \\
& \hat{v}_{3}(L, L)=\Phi_{c} \hat{v}_{3}(L-1, L-1)+G_{3}(L)(y(L) \\
& -\left(H \Phi \hat{x}(L-1, L-1)+\left(\Phi_{c}\right)^{2} \hat{v}_{2}(L-1, L-1)\right. \\
& \left.\left.+\Phi_{c} \hat{v}_{3}(L-1, L-1)\right)\right), \\
& \hat{v}_{3}(0,0)=0
\end{aligned}
$$

Filtering variance function of $\hat{x}(L, L): S_{11}(L)$

$$
\begin{aligned}
& S_{11}(L)=E\left[\hat{x}(L, L) \hat{x}^{T}(L, L)\right] \\
& S_{11}(L)=\Phi S_{11}(L-1) \Phi^{T} \\
& +G_{1}(L)\left(H K_{x}(L, L)-\left(H \Phi S_{11}(L-1) \Phi^{T}\right.\right. \\
& \left.\left.+\left(\Phi_{c}\right)^{2} S_{21}(L-1) \Phi^{T}+\Phi_{c} S_{31}(L-1) \Phi^{T}\right)\right), \\
& S_{11}(0)=0
\end{aligned}
$$

Cross-variance function of $\hat{x}(L, L)$ with $\hat{v}_{2}(L, L)$ : $S_{12}(L)=E\left[\hat{x}(L, L) \hat{v}_{2}^{T}(L, L)\right]$

$$
\begin{aligned}
& S_{12}(L)=\Phi S_{12}(L-1) \Phi_{c}^{T} \\
& +G_{1}(L)\left(\Phi_{c} K_{c}(L, L)-\left(H \Phi S_{12}(L-1) \Phi_{c}^{T}\right.\right. \\
& \left.\left.+\left(\Phi_{c}\right)^{2} S_{22}(L-1) \Phi_{c}^{T}+\Phi_{c} S_{32}(L-1) \Phi_{c}^{T}\right)\right), \\
& S_{12}(0)=0
\end{aligned}
$$




$$
\begin{aligned}
& G_{1}(L)=\left[K_{x}(L, L) H^{T}-\Phi S_{11}(L-1) \Phi^{T} H^{T}\right. \\
& \left.-\Phi S_{12}(L-1)\left(\Phi_{c}^{T}\right)^{2}-\Phi S_{13}(L-1) \Phi_{c}^{T}\right] \\
& \times\left[R_{u}(L)+\left(H K_{x}(L, L)-\left(H \Phi S_{11}(L-1) \Phi^{T}\right.\right.\right. \\
& \left.\left.+\left(\Phi_{c}\right)^{2} S_{21}(L-1) \Phi^{T}+\Phi_{c} S_{31}(L-1) \Phi^{T}\right)\right) H^{T} \\
& +\left(\Phi_{c} K_{c}(L, L)-\left(H \Phi S_{12}(L-1) \Phi_{c}^{T}\right.\right. \\
& \left.\left.+\left(\Phi_{c}\right)^{2} S_{22}(L-1) \Phi_{c}^{T}+\Phi_{c} S_{32}(L-1) \Phi_{c}^{T}\right)\right) \Phi_{c}^{T} \\
& +\left(R_{u}(L)-\left(H \Phi S_{13}(L-1) \Phi_{c}^{T}\right.\right. \\
& \left.\left.\left.+\left(\Phi_{c}\right)^{2} S_{23}(L-1) \Phi_{c}^{T}+\Phi_{c} S_{33}(L-1) \Phi_{c}^{T}\right)\right)\right]^{-1}
\end{aligned}
$$

Filter gain for $\hat{v}_{2}(L, L): G_{2}(L)$

$$
\begin{aligned}
& G_{2}(L)=\left[K_{c}(L, L) \Phi_{c}^{T}-\Phi_{c} S_{21}(L-1) \Phi^{T} H^{T}\right. \\
& \left.-\Phi_{c} S_{22}(L-1)\left(\Phi_{c}^{T}\right)^{2}-\Phi_{c} S_{23}(L-1) \Phi_{c}^{T}\right] \\
& \times\left[R_{u}(L)+\left(H K_{x}(L, L)-\left(H \Phi S_{11}(L-1) \Phi^{T}\right.\right.\right. \\
& \left.\left.+\left(\Phi_{c}\right)^{2} S_{21}(L-1) \Phi^{T}+\Phi_{c} S_{31}(L-1) \Phi^{T}\right)\right) H^{T} \\
& +\left(\Phi_{c} K_{c}(L, L)-\left(H \Phi S_{12}(L-1) \Phi_{c}^{T}\right.\right. \\
& \left.\left.+\left(\Phi_{c}\right)^{2} S_{22}(L-1) \Phi_{c}^{T}+\Phi_{c} S_{32}(L-1) \Phi_{c}^{T}\right)\right) \Phi_{c}^{T} \\
& +\left(R_{u}(L)-\left(H \Phi S_{13}(L-1) \Phi_{c}^{T}\right.\right. \\
& \left.\left.\left.+\left(\Phi_{c}\right)^{2} S_{23}(L-1) \Phi_{c}^{T}+\Phi_{c} S_{33}(L-1) \Phi_{c}^{T}\right)\right)\right]^{-1}
\end{aligned}
$$

Filter gain for $\hat{v}_{3}(L, L): G_{3}(L)$

$$
\begin{aligned}
& G_{3}(L)=\left[R_{u}(L)-\Phi_{c} S_{31}(L-1) \Phi^{T} H^{T}\right. \\
& \left.-\Phi_{c} S_{32}(L-1)\left(\Phi_{c}^{T}\right)^{2}-\Phi_{c} S_{33}(L-1) \Phi_{c}^{T}\right] \\
& \times\left[R_{u}(L)+\left(H K_{x}(L, L)-\left(H \Phi S_{11}(L-1) \Phi^{T}\right.\right.\right. \\
& \left.\left.+\left(\Phi_{c}\right)^{2} S_{21}(L-1) \Phi^{T}+\Phi_{c} S_{31}(L-1) \Phi^{T}\right)\right) H^{T} \\
& +\left(\Phi_{c} K_{c}(L, L)-\left(H \Phi S_{12}(L-1) \Phi_{c}^{T}\right.\right. \\
& \left.\left.+\left(\Phi_{c}\right)^{2} S_{22}(L-1) \Phi_{c}^{T}+\Phi_{c} S_{32}(L-1) \Phi_{c}^{T}\right)\right) \Phi_{c}^{T} \\
& +\left(R_{u}(L)-\left(H \Phi S_{13}(L-1) \Phi_{c}^{T}\right.\right. \\
& \left.\left.\left.+\left(\Phi_{c}\right)^{2} S_{23}(L-1) \Phi_{c}^{T}+\Phi_{c} S_{33}(L-1) \Phi_{c}^{T}\right)\right)\right]^{-1}
\end{aligned}
$$

Proof of Theorem $\mathbf{1}$ is deferred to the Appendix.

From Theorem 1, the filtering error variance function $\tilde{P}_{F}(L, L)$ and the prediction error variance function $\widetilde{P}_{P}(L, L-1)$, for the signal $z(L)$, are given by

$$
\begin{aligned}
& \widetilde{P}_{F}(L, L)=K_{z}(L, L)-H S_{11}(L) H^{T}, \\
& \tilde{P}_{P}(L, L-1)=K_{z}(L, L)-H \Phi S_{11}(L-1) \Phi^{T} H^{T} .
\end{aligned}
$$

where $K_{z}(L, L)$ represents the variance function of the signal $z(L)$.

According to the smoothing and filtering algorithms in Theorem 1, the calculation steps for the fixedinterval smoothing estimate $\hat{z}(k, L)$ of the signal $z(k)$ can be shown in the flowchart of Fig. 1.

\section{A Numerical Simulation Example}

In this section, to show the efficiency of the estimation characteristics of the proposed RLS Wiener fixed-interval smoothing algorithm, a numerical example is demonstrated. The fixed-interval s moothing estimate is calculated according to the flowchart in Fig. 1.

Let a scalar observation equation be described by

$$
y(k)=z(k)+v_{c}(k), z(k)=H x(k) .
$$

Here, $v_{c}(k)$ is the zero-mean colored observation noise. Let the signal $z^{(k)}$ be generated by the secondorder AR model.

$$
\begin{aligned}
& z(k+1)=-a_{1} z(k)-a_{2} z(k-1)+w(k), \\
& E[w(k) w(s)]=\sigma^{2} \delta_{K}(k-s), \\
& a_{1}=-0.1, \quad a_{2}=-0.8, \quad \sigma=0.5 .
\end{aligned}
$$

The corresponding state-space model for $z(k)$ can be written as

$$
\begin{aligned}
& z(k)=H x(k)=x_{1}(k), \quad H=\left[\begin{array}{ll}
1 & 0
\end{array}\right], \quad x(k)=\left[\begin{array}{l}
x_{1}(k) \\
x_{2}(k)
\end{array}\right], \\
& {\left[\begin{array}{c}
x_{1}(k+1) \\
x_{2}(k+1)
\end{array}\right]=\left[\begin{array}{cc}
0 & 1 \\
-a_{2} & -a_{1}
\end{array}\right]\left[\begin{array}{l}
x_{1}(k) \\
x_{2}(k)
\end{array}\right]+\left[\begin{array}{l}
0 \\
1
\end{array}\right] w(k) .}
\end{aligned}
$$

The auto-covariance function of the signal $z(k)$ is described as follows [13]:

$$
\begin{aligned}
& K(0)=\sigma^{2}, \\
& K(m)=\sigma^{2}\left\{\alpha_{1}\left(\alpha_{2}^{2}-1\right) \alpha_{1}^{m} /\left[\left(\alpha_{2}-\alpha_{1}\right)\left(\alpha_{2} \alpha_{1}+1\right)\right]\right. \\
& \left.-\alpha_{2}\left(\alpha_{1}^{2}-1\right) \alpha_{2}^{m} /\left[\left(\alpha_{2}-\alpha_{1}\right)\left(\alpha_{2} \alpha_{1}+1\right)\right]\right\}, \\
& 0<m, \quad \alpha_{1}, \alpha_{2}=\left(-a_{1} \pm \sqrt{a_{1}^{2}-4 a_{2}}\right) / 2 .
\end{aligned}
$$

From (49) and (50), it can be found that

$$
\begin{aligned}
& K_{x}(k, k)=\left[\begin{array}{cc}
K(0) & K(1) \\
K(1) & K(0)
\end{array}\right], \quad \Phi=\left[\begin{array}{cc}
0 & 1 \\
-a_{2} & -a_{1}
\end{array}\right], \\
& K(0)=0.25, \quad K(1)=0.125 .
\end{aligned}
$$

Let the state equation for $v_{c}(k)$ be given by

$$
\begin{aligned}
& v_{c}(k+1)=\Phi_{c} v_{c}(k)+u(k), \\
& E\left[u(k) u^{T}(s)\right]=R_{u} \delta_{K}(k-s), \\
& \Phi_{c}=0.91, \quad R_{u}=0.01,
\end{aligned}
$$

where $u(k)$ is the white input noise in the state equation (52) for the colored observation noise process. The autovariance function of the colored observation noise $v_{c}(\cdot) \quad$ satisfies the relationships $K_{c}(k+1, k+1)=K_{c}(k, k)=K_{c}(0)$ and hence, this lead to $K_{c}(0)=\frac{R_{u}}{1-a^{2}} \quad$ in wide-sense stationary stochastic systems. 


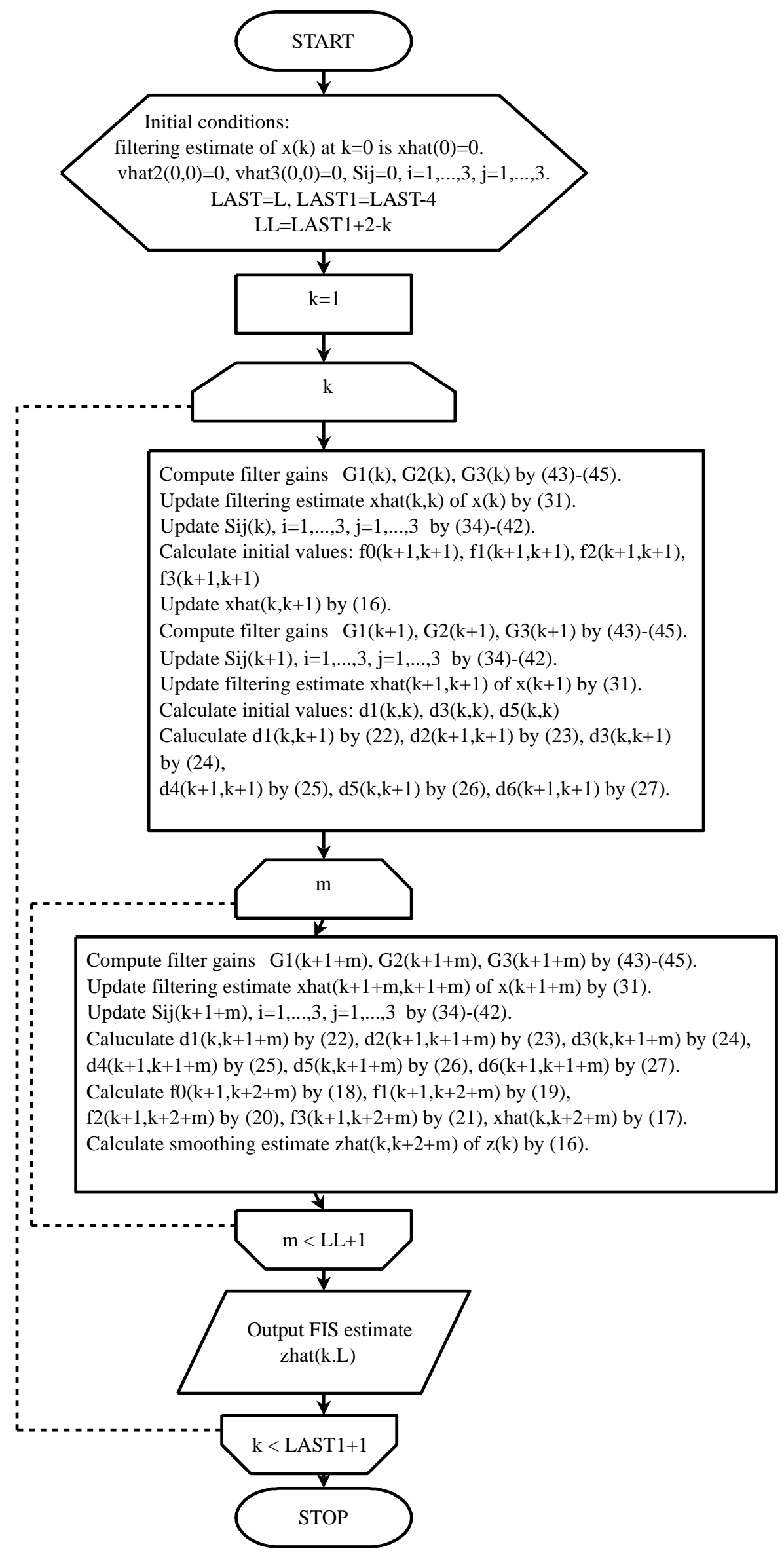

Fig. 1: Flowchart for the fixed-interval smoothing estimate $\hat{z}(k, L)$. 
Substituting $H, \Phi, K_{x}(L, L)=K_{x}(0), \Phi_{c}$, $K_{c}(L, L)=K_{c}(0)$ and $R_{u}$ into the RLS Wiener estimation algorithms of Theorem 1, the fixed-interval smoothing and filtering estimates are calculated.

Fig. 2 illustrates the colored observation noise process $v_{c}(k)$ vs. $k, 1 \leq k \leq 500$, for the variance $R_{u}=0.01$ in (52). Fig. 3 illustrates the fixed-interval smoothing estimate $\hat{z}(k, L), L=500$, vs. $k, 1 \leq k \leq 500$, for the variance $R_{u}=0.01$. Fig. 4 illustrates the MSVs of the filtering errors $z(L)-\hat{z}(L, L)$ of the signal $z(L)$ and the fixed-interval smoothing errors $z(k)-\hat{z}(k, L)$ of $z(k)$ vs. vs. $L$ for $R_{u}=0.001\left(K_{c}(0)=0.0582\right)$. In Fig. 4 , as the fixed interval $L$ increases, $100 \leq L \leq 600$, the MSV of the fixed-interval s moothing errors gradually decreases. Also, for $100 \leq L \leq 700$, the MSV of the fixed-interval s moothing errors $z(k)-\hat{z}(k, L)$ is less than the MSV of the filtering errors $z(k)-\hat{z}(k, k)$, $1 \leq k \leq L$. Hence, the estimation accuracy of the fixedinterval smoother is better than that of the filter for $100 \leq L \leq 700$. From this fact, the fixed-interval smoothing algorithm, calculated based on the flowchart of Fig. 1, is correct.

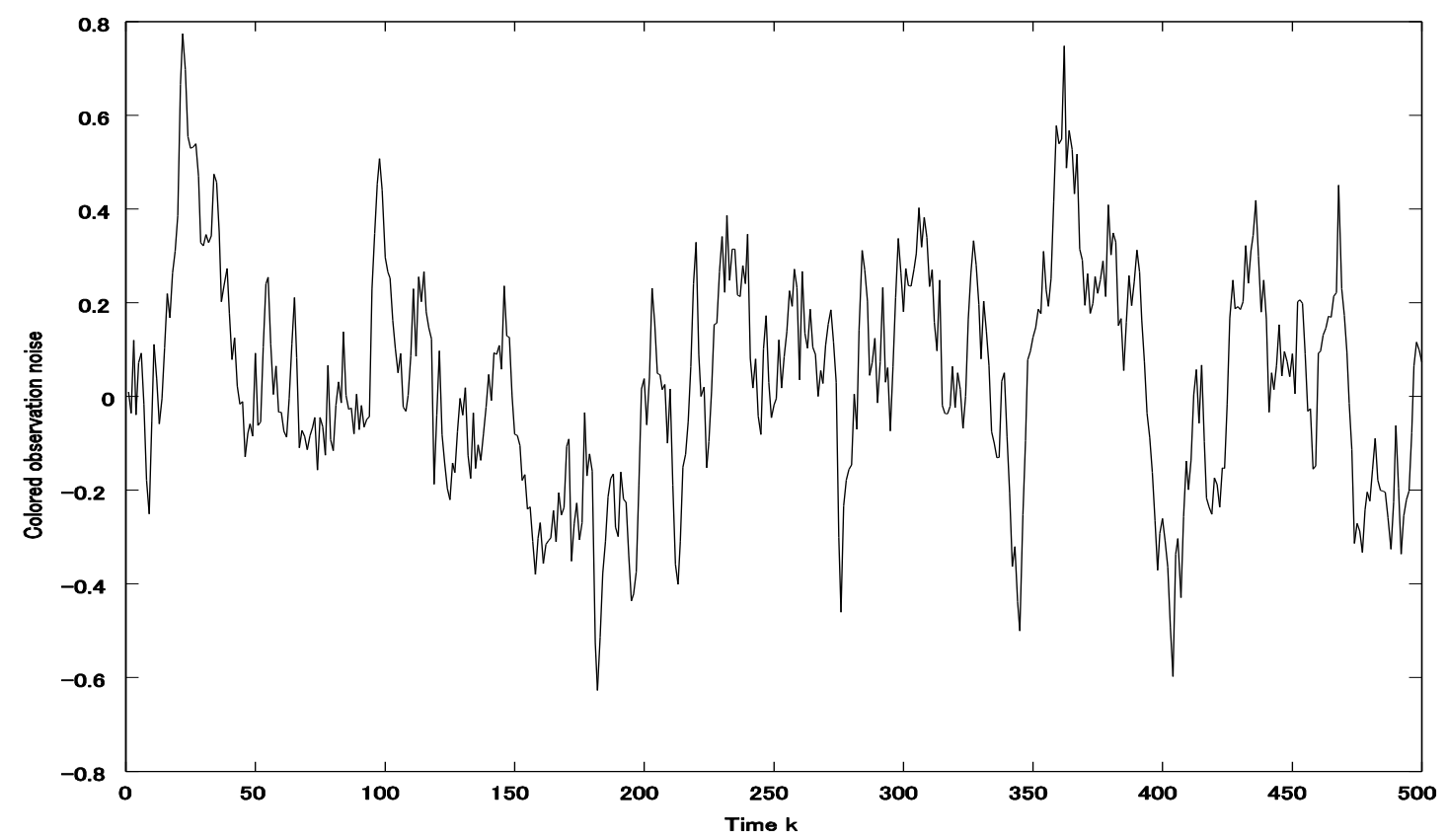

Fig. 2: Colored observation noise process $v_{c}(k)$ vs. $k, 1 \leq k \leq 500$, for the variance $R_{u}=0.01$ in (52).

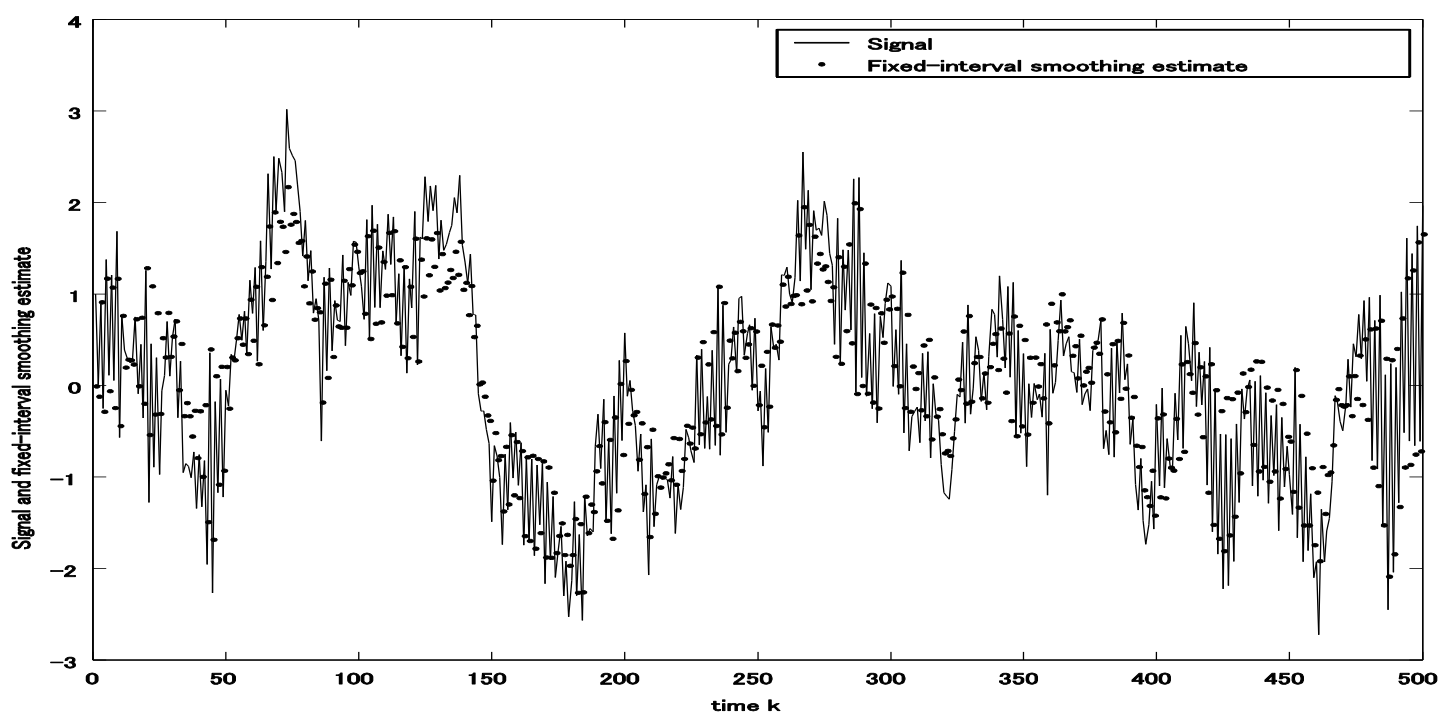

Fig. 3: Fixed-interval smoothing estimate $\hat{z}(k, L), L=500$, vs. $k$ for the variance $R_{u}=0.01$. 


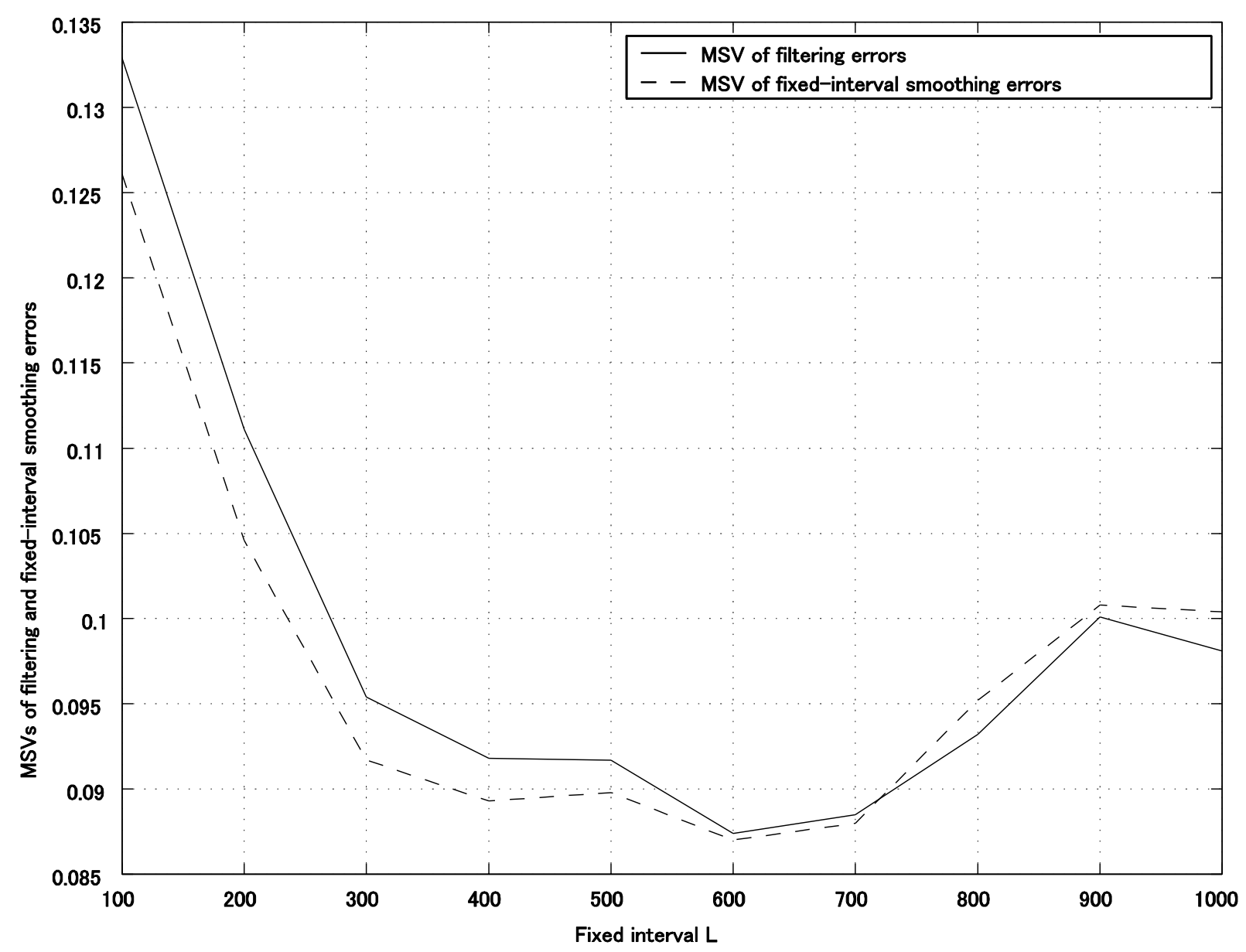

Fig. 4: MSVs of the filtering errors $z(k)-\hat{z}(k, k), 1 \leq k \leq L$ and the fixed-interval smoothing errors $z(k)-\hat{z}(k, L)$ vs. $L, 100 \leq L \leq 1000$.

\section{Conclusions}

In this paper, the RLS W iener smoothing problems, with relation to the innovation theory, have been considered for the colored observation noise.

A numerical simulation example has shown that the proposed estimation algorithms for the RLS Wiener fixed-interval smoothing and filtering estimates, in the case of the colored observation noise, are feasible. In Fig. 4, as the fixed interval $L$ increases, $100 \leq L \leq 600$, the MSV of the fixed-interval smoothing errors gradually decreases. Similar tendency, on the estimation accuracy, also fit to the RLS Wiener filter. Also, for $100 \leq L \leq 700$, the MSV of the fixed-interval smoothing errors $z(k)-\hat{z}(k, L)$ is less than the MSV of the filtering errors $z(k)-\hat{z}(k, k), 1 \leq k \leq L$. Hence, for $100 \leq L \leq 700$, the estimation accuracy of the RLS Wiener fixedinterval smoother is superior to that of the RLS Wiener filter for the colored observation noise.

The RLS Wiener estimators do not use the information of the variance $Q(k)$, for the input noise $w(k)$, and the input matrix $G$ in the state equation (4), in comparison with the Kalman estimation technique [13]. In the RLS Wiener estimators, it is not necessary to take account of the degraded estimation accuracy caused by the modeling errors for $Q(k)$ and $G$.

\section{References}

[1] Nakamori S. Recursive estimation technique of signal from output measurement data in linear discrete-time systems [J]. IEICE Trans. Fundamentals, 1995, E-78-A: 600-607.

[2] Nakamori S. Chandrasekhar-type recursive Wiener estimation technique in linear discrete-time systems [J]. Applied Mathematics and Computation, 2007, 188: 1656-1665.

[3] Nakamori S. Square-root algorithms of RLS Wiener filter and fixed-point smoother in linear discrete stochastic systems [J]. Applied Mathematics and Computation, 2008, 203(1): 186193.

[4] Nakamori S. Design of RLS Wiener FIR filter using covariance information in linear discretetime stochastic systems [J]. Digital Signal Processing, 2010, .20(5): 1310-1329. 
[5] Boll S. Suppression of acoustic noise in speech using spectral subtraction [J]. IEEE Trans. Acoustics, Speech and Signal Processing, 1979, ASSP-27(2): 113-120.

[6] Xiong S. S., Zhou Z. Y. Neural filtering of colored noise based on Kalman filter structure [J]. IEEE Transactions on Instrumentation and Measurement, 2003, 52(3): 742-747.

[7] Simon D. Optimal state estimation: Kalman, H infinity, and nonlinear approaches [M]. John Wiley \& Sons, New York, N Y, 2006.

[8] Must'iere F., Boli'c M., Bouchad M. Improved colored noise handling in Kalman filter-based speech enhancement algorithms [C]. In: Canadian Conference on Electrical Computer Engineering, 2008, CCECE 2008, 497-500.

[9] Park S., Choi S. A constrained sequential EM algorithm for speech enhancement [J]. Neural Networks [J]. 2008, 21: 1401-1409.

[10] Shuli Sun Reduced-order Wiener state estimators for descriptor system with multi-observation lags and MA colored observation noise [C]. In: Control Conference 2008, CCC 2008, $27^{\text {th }}$ Chinese, 2008, 417-420..

[11] Nakamori S. Estimation of signal and parameters using covariance information in linear continuous systems [J]. Mathematical and Computer modeling, 1992, 16(10): 3-15.

[12] Mahmoudi A., Karimi M. Parameter estimation of autoregressive signals from observations corrupted with colored noise [J]. Signal Processing, 2010, 90(1): 157-164.

[13] Nakamori S. Design of RLS W iener smoother and filter for colored observation noise in linear discrete-time stochastic systems [J]. J. of Signal and Information Processing, 2012, 3(3): 316-329.

[14] Kailath T. Lectures on Wiener and Kalman filtering [M]. CISM Monographs, No. 140, Springer-Verlag, New York, N Y, 1981.

[15] Kailath T., Frost P. An innovations approach to least-squares estimation Part II: Linear s moothing in additive white noise [J]. IEEE Trans. Automatic Control, 1968, AC-13(6): 655-660.

[16] Sage A. P., Melsa J. L. Estimation theory with applications to communications and control [M]. McGraw-Hill, New York, N Y, 1971.

\section{Appendix Proof of Theorem 1}

From the equation for the filter gain (36) in [13], $E\left[x(k) v^{T}(k)\right]$ is given by

$$
\begin{aligned}
& E\left[x(k) v^{T}(k)\right]=K_{x}(k, k) H^{T}-\Phi S_{11}(k-1)-\Phi S_{12}(k-1)\left(\Phi_{c}^{T}\right)^{2} \\
& -\Phi S_{13}(k-1) \Phi_{c}^{T} .
\end{aligned}
$$

From (15) together with (13), it is seen that

$$
\begin{aligned}
& g(k, s) \Delta(s)=E\left[x(k) v^{T}(s)\right] \\
& =E\left[x ( k ) \left(y(s)-H \Phi \hat{x}(s-1, s-1)-\Phi_{c}^{2} \hat{v}_{2}(s-1, s-1)\right.\right. \\
& \left.\left.-\Phi_{c} \hat{v}_{3}(s-1, s-1)\right)^{T}\right], \quad 1 \leq k<s .
\end{aligned}
$$

Substituting (A-1) into (8), (8) can be reduced to

$\hat{x}(k, L)=\hat{x}(k, k)+\sum_{i=k+1}^{L}\left\{K_{x}(k, i) H^{T}-E\left[x(k) \hat{x}^{T}(i-1, i-1)\right] \Phi^{T} H^{T}\right.$

$\left.E\left[x(k) \hat{v}_{2}^{T}(i-1, i-1)\right]\left(\Phi_{c}^{T}\right)^{2}-E\left[x(k) \hat{v}_{3}^{T}(i-1, i-1)\right] \Phi_{c}^{T}\right\} \Delta^{-1}(i) v(i)$

$=\hat{x}(k, k)+f_{0}(k+1, L)-f_{1}(k+1, L)-f_{2}(k+1, L)-f_{3}(k+1, L)$.

Here, $f_{0}(k+1, L) \quad, \quad f_{1}(k+1, L) \quad, \quad f_{2}(k+1, L) \quad$ and $f_{3}(k+1, L)$ are given by

$$
\begin{aligned}
& f_{0}(k+1, L)=\sum_{i=k+1}^{L} K_{x}(k, i) H^{T} \Delta^{-1}(i) v(i), \\
& f_{1}(k+1, L)=\sum_{i=k+1}^{L} E\left[x(k) \hat{x}^{T}(i-1, i-1)\right] \Phi^{T} H^{T} \Delta^{-1}(i) v(i), \\
& f_{2}(k+1, L)=\sum_{i=k+1}^{L} E\left[x(k) \hat{v}_{2}^{T}(i-1, i-1)\right]\left(\Phi_{c}^{T}\right)^{2} \Delta^{-1}(i) v(i), \\
& f_{2}(k+1, L)=\sum_{i=k+1}^{L} E\left[x(k) \hat{v}_{3}^{T}(i-1, i-1)\right] \Phi_{c}^{T} \Delta^{-1}(i) v(i) .
\end{aligned}
$$

Using (3) and introducing a function $M(L)$ given by

$$
\begin{aligned}
& M(L)=\sum_{i=k+1}^{L} A^{T}(i) H^{T} \Delta^{-1}(i) v(i) \\
& f_{0}(k+1, L) \text { is written as } \\
& f_{0}(k+1, L)=\sum_{i=k+1}^{L} B(k) A^{T}(i) H^{T} \Delta^{-1}(i) v(i) \\
& =K_{x}(k, k)\left(\Phi^{T}\right)^{-k} M(L) .
\end{aligned}
$$

From the equation for updating $M(L)$

$$
M(L)=M(L-1)+A^{T}(L) H^{T} \Delta^{-1}(L) v(L),
$$

(18) is obtained. Initial value for $f_{0}(k+1, L)$, at $L=k+1$, is $f_{0}(k+1, k+1)$, which is derived as follows:

$$
\begin{aligned}
& f_{0}(k+1, k+1)=K_{x}(k, k+1) H^{T} \Delta^{-1}(k+1) v(k+1) \\
& =K_{x}(k, k) \Phi^{T} H^{T} \Delta^{-1}(k+1) v(k+1) .
\end{aligned}
$$

Subtracting $f_{1}(k+1, L-1)$ from $f_{1}(k+1, L)$, it can be shown that

$$
\begin{aligned}
& f_{1}(k+1, L)-f_{1}(k+1, L-1)=E\left[x(k) \hat{x}^{T}(L-1, L-1)\right] \\
& \times \Phi^{T} H^{T} \Delta^{-1}(L) v(L) .
\end{aligned}
$$

Upon substituting (A-57) and (A-58), presented in [13], (A-2) can be reduced to 
$f_{1}(k+1, L)-f_{1}(k+1, L-1)=E\left[x(k) O_{1}^{T}(L-1)\right]\left(\Phi^{T}\right)^{L-1}$

$\times \Phi^{T} H^{T} \Delta^{-1}(L) \cup(L)$

$=\sum_{j=1}^{L-1} E\left[x(k) y^{T}(j)\right] J_{1}^{T}(j, L-1)\left(\Phi^{T}\right)^{L} H^{T} \Delta^{-1}(L) v(L)$

$=\sum_{j=1}^{k} A(k) B^{T}(j) H^{T} J_{1}^{T}(j, L-1)\left(\Phi^{T}\right)^{L} H^{T} \Delta^{-1}(L) v(L)$

$+\sum_{j=k+1}^{L-1} B(k) A^{T}(j) H^{T} J_{1}^{T}(j, L-1)\left(\Phi^{T}\right)^{L} H^{T} \Delta^{-1}(L) v(L)$

$=A(k) l_{1}^{T}(k, L-1)\left(\Phi^{T}\right)^{L} H^{T} \Delta^{-1}(L) v(L)$

$+B(k) l_{2}^{T}(k+1, L-1)\left(\Phi^{T}\right)^{L} H^{T} \Delta^{-1}(L) v(L)$,

where

$$
\begin{aligned}
& l_{1}(k, L-1)=\sum_{i=1}^{k} J_{1}(i, L-1) H B(i), \\
& l_{2}(k+1, L)=\sum_{i=k+1}^{L} J_{1}(i, L) H A(i) .
\end{aligned}
$$

Introducing functions

$$
\begin{aligned}
& d_{1}(k, L-1)=\Phi^{L} l_{1}(k, L-1) A^{T}(k), \\
& d_{2}(k+1, L-1)=\Phi^{L} l_{2}(k+1, L-1) B^{T}(k),
\end{aligned}
$$

(19), for updating $f_{1}(k+1, L)$, is obtained. Initial value in (19) for $f_{1}(k+1, L)$, at $L=k+1$, is $f_{1}(k+1, k+1)$, which is derived, from (A-14), (A-37), (A-57) and (A-58) presented in [13], as follows:

$f_{1}(k+1, k+1)=E\left[x(k) \hat{x}^{T}(k, k)\right] \Phi^{T} H^{T} \Delta^{-1}(k+1) v(k+1)$

$=E\left[x(k) O_{1}^{T}(k)\right]\left(\Phi^{T}\right)^{k} \Phi^{T} H^{T} \Delta^{-1}(k+1) v(k+1)$

$=\sum_{i=1}^{k} A(k) B^{T}(i) H^{T} J_{1}^{T}(i, k)\left(\Phi^{T}\right)^{k+1} H^{T} \Delta^{-1}(k+1) v(k+1)$

$=A(k) r_{11}^{T}(k)\left(\Phi^{T}\right)^{k} \Phi^{T} H^{T} \Delta^{-1}(k+1) v(k+1)$

$=S_{11}^{T}(k) \Phi^{T} H^{T} \Delta^{-1}(k+1) v(k+1)$.

Subtracting $f_{2}(k+1, L-1)$ from $f_{2}(k+1, L)$, it can be shown that

$$
\begin{aligned}
& f_{2}(k+1, L)-f_{2}(k+1, L-1)=E\left[x(k) \hat{v}_{2}^{T}(L-1, L-1)\right] \\
& \times\left(\Phi_{c}^{T}\right)^{2} \Delta^{-1}(L) v(L) .
\end{aligned}
$$

Substituting (A-60) and (A-61), derived in [13], into (A-4), it can be reduced to

$$
\begin{aligned}
& f_{2}(k+1, L)-f_{2}(k+1, L-1)=E\left[x(k) O_{2}^{T}(L-1)\right] \\
\times & \left(\Phi_{c}^{T}\right)^{L-1}\left(\Phi_{c}^{T}\right)^{2} \Delta^{-1}(L) v(L) \\
= & \sum_{j=1}^{L-1} E\left[x(k) y^{T}(j)\right] J_{2}^{T}(j, L-1)\left(\Phi_{c}^{T}\right)^{L+1} \Delta^{-1}(L) v(L) \\
= & \sum_{j=1}^{k} A(k) B^{T}(j) H^{T} J_{2}^{T}(j, L-1)\left(\Phi_{c}^{T}\right)^{L+1} \Delta^{-1}(L) v(L) \\
+ & \sum_{j=k+1}^{L-1} B(k) A^{T}(j) H^{T} J_{2}^{T}(j, L-1)\left(\Phi_{c}^{T}\right)^{L+1} \Delta^{-1}(L) v(L) \\
= & A(k) l_{3}^{T}(k, L-1)\left(\Phi_{c}^{T}\right)^{L+1} \Delta^{-1}(L) v(L) \\
+ & B(k) l_{4}^{T}(k+1, L-1)\left(\Phi_{c}^{T}\right)^{L+1} \Delta^{-1}(L) v(L),
\end{aligned}
$$

where

$$
\begin{aligned}
& l_{3}(k, L-1)=\sum_{i=1}^{k} J_{2}(i, L-1) H B(i), \\
& l_{4}(k+1, L-1)=\sum_{i=k+1}^{L-1} J_{2}(i, L-1) H A(i) .
\end{aligned}
$$

Introducing functions

$$
\begin{aligned}
& d_{3}(k, L)=\Phi_{c}^{L+2} l_{3}(k, L) A^{T}(k), \\
& d_{4}(k+1, L)=\Phi_{c}^{L+2} l_{4}(k+1, L) B^{T}(k),
\end{aligned}
$$

(20), for updating $f_{2}(k+1, L)$, is obtained. In itial value in (20) for $f_{2}(k+1, L)$, at $L=k+1$, is $f_{2}(k+1, k+1)$, which is derived, from (A-19), (A-37), (A-60) and (A-61) presented in [13], as follows:

$$
\begin{aligned}
& f_{2}(k+1, k+1)=E\left[x(k) \hat{v}_{2}^{T}(k, k)\right]\left(\Phi_{c}^{T}\right)^{2} \Delta^{-1}(k+1) v(k 2+1) \\
& =E\left[x(k) O_{2}^{T}(k)\right]\left(\Phi_{c}^{T}\right)^{k}\left(\Phi_{c}^{T}\right)^{2} \Delta^{-1}(k+1) v(k+1) \\
& =\sum_{i=1}^{k} A(k) B^{T}(i) H^{T} J_{2}^{T}(i, k)\left(\Phi_{c}^{T}\right)^{k+2} \Delta^{-1}(k+1) v(k+1) \\
& =A(k) r_{21}^{T}(k)\left(\Phi_{c}^{T}\right)^{k+2} \Delta^{-1}(k+1) v(k+1) \\
& =S_{21}^{T}(k)\left(\Phi_{c}^{T}\right)^{2} \Delta^{-1}(k+1) v(k+1)
\end{aligned}
$$

Subtracting $f_{3}(k+1, L-1)$ from $f_{3}(k+1, L)$, it can be shown that

$$
\begin{aligned}
& f_{3}(k+1, L)-f_{3}(k+1, L-1) \\
& =E\left[x(k) \hat{v}_{3}^{T}(L-1, L-1)\right] \Phi_{c}^{T} \Delta^{-1}(L) v(L) .
\end{aligned}
$$

Substituting (A-60) and (A-61), presented in [13], into (A-6), it can be reduced to

$$
\begin{aligned}
& f_{3}(k+1, L)-f_{3}(k+1, L-1)=E\left[x(k) O_{3}^{T}(L-1)\right] \\
\times & \left(\Phi_{c}^{T}\right)^{L-1}\left(\Phi_{c}^{T}\right) \Delta^{-1}(L) v(L) \\
= & \sum_{j=1}^{L-1} E\left[x(k) y^{T}(j)\right] J_{3}^{T}(j, L-1)\left(\Phi_{c}^{T}\right)^{L} \Delta^{-1}(L) v(L) \\
= & \sum_{j=1}^{k} A(k) B^{T}(j) H^{T} J_{3}^{T}(j, L-1)\left(\Phi_{c}^{T}\right)^{L} \Delta^{-1}(L) v(L) \\
+ & \sum_{j=k+1}^{L-1} B(k) A^{T}(j) H^{T} J_{3}^{T}(j, L-1)\left(\Phi_{c}^{T}\right)^{L} \Delta^{-1}(L) v(L) \\
= & A(k) l_{5}^{T}(k, L-1)\left(\Phi_{c}^{T}\right)^{L} \Delta^{-1}(L) v(L) \\
+ & B(k) l_{6}^{T}(k+1, L-1)\left(\Phi_{c}^{T}\right)^{L} \Delta^{-1}(L) v(L),
\end{aligned}
$$

where

$$
\begin{aligned}
& l_{5}(k, L-1)=\sum_{i=1}^{k} J_{3}(i, L-1) H B(i), \\
& l_{6}(k+1, L-1)=\sum_{i=k+1}^{L-1} J_{3}(i, L-1) H A(i) .
\end{aligned}
$$

Introducing functions

$$
\begin{aligned}
& d_{5}(k, L)=\Phi_{c}^{L} l_{5}(k, L) A^{T}(k), \\
& d_{6}(k+1, L-1)=\Phi_{c}^{L} l_{6}(k+1, L-1) B^{T}(k),
\end{aligned}
$$


We obtain (21) for updating $f_{3}(k+1, L)$. Initial value in (21) for $f_{3}(k+1, L)$, at $L=k+1$, is $f_{3}(k+1, k+1)$, which is derived, from (A-24), (A-37), (A-60) and (A61) presented in [13], as follows:

$$
\begin{aligned}
& f_{3}(k+1, k+1)=E\left[x(k) \hat{v}_{3}^{T}(k, k)\right] \Phi_{c}^{T} \Delta^{-1}(k+1) v(k 2+1) \\
& =E\left[x(k) O_{3}^{T}(k)\right]\left(\Phi_{c}^{T}\right)^{k} \Phi_{c}^{T} \Delta^{-1}(k+1) v(k+1) \\
& =\sum_{i=1}^{k} A(k) B^{T}(i) H^{T} J_{3}^{T}(i, k)\left(\Phi_{c}^{T}\right)^{k+1} \Delta^{-1}(k+1) v(k+1) \\
& =A(k) r_{31}^{T}(k)\left(\Phi_{c}^{T}\right)^{k+1} \Delta^{-1}(k+1) v(k+1) \\
& =S_{31}^{T}(k) \Phi_{c}^{T} \Delta^{-1}(k+1) v(k+1) .
\end{aligned}
$$

Subtracting $l_{1}(k, L-1)$ from $l_{1}(k, L)$, it can be shown that

$$
\begin{aligned}
& l_{1}(k, L)-l_{1}(k, L-1)=\sum_{i=1}^{k}\left(J_{1}(i, L)\right. \\
& \left.-J_{1}(i, L-1)\right) H B(i) \\
& =-J_{1}(L, L)\left(H \Phi^{L} l_{1}(k, L-1)+\Phi_{c}^{L+1} l_{3}(k, L-1)\right. \\
& \left.+\Phi_{c}^{L} l_{5}(k, L-1)\right) .
\end{aligned}
$$

Substituting (A-8) into the equation for $d_{1}(k, L)$, it can be reduced to

$$
\begin{aligned}
& d_{1}(k, L)=\Phi^{L+1}\left\{l_{1}(k, L-1)-J_{1}(L, L)\left(H \Phi^{L} l_{1}(k, L-1)\right.\right. \\
& \left.\left.+\Phi_{c}^{L+1} l_{3}(k, L-1)+\Phi_{c}^{L} l_{5}(k, L-1)\right)\right\} A^{T}(k) \\
& =\Phi_{c} d_{1}(k, L-1)-\Phi G_{1}(L)\left(H d_{1}(k, L-1)\right. \\
& \left.+d_{3}(k, L-1)+d_{5}(k, L-1)\right),
\end{aligned}
$$

where we used the expression for the filter gain $G_{1}(L)=\Phi^{L} J_{1}(L, L)$ in [13]. The initial value for $d_{1}(k, L)$, at $L=k$, is $d_{1}(k, k)$, which is derived as follows:

$$
\begin{aligned}
& d_{1}(k, k)=\Phi^{k+1} l_{1}(k, k) A^{T}(k) \\
& =\Phi^{k+1} \sum_{i=1}^{k} J_{1}(i, k) H B(i) A^{T}(k) \\
& =\Phi^{k+1} r_{11}(k)\left(\Phi^{T}\right)^{k} \\
& =\Phi S_{11}(k) .
\end{aligned}
$$

Here, (A-14) and (A-37), presented in [13], are used.

Subtracting $l_{2}(k+1, L-1)$ from $l_{2}(k+1, L)$, it can be shown that

$$
\begin{aligned}
& l_{2}(k+1, L)-l_{2}(k+1, L-1)=J_{1}(L, L) H A(L) \\
& +\sum_{i=k+1}^{L-1}\left(J_{1}(i, L)-J_{1}(i, L-1)\right) H A(i) \\
& =J_{1}(L, L) H A(L)-J_{1}(L, L)\left(H \Phi^{L} l_{2}(k+1, L-1)\right. \\
& \left.+\Phi_{c}^{L+1} l_{4}(k+1, L-1)+\Phi_{c}^{L} l_{6}(k+1, L-1)\right) .
\end{aligned}
$$

Substituting (A-9) into the equation for $d_{2}(k+1, L)$, it can be reduced to

$$
\begin{aligned}
& d_{2}(k+1, L)=\Phi^{L+1}\left\{l_{2}(k+1, L-1)+J_{1}(L, L)(H A(L)\right. \\
& -H \Phi^{L} l_{2}(k+1, L-1)-\Phi_{c}^{L+1} l_{4}(k+1, L-1) \\
& \left.\left.-\Phi_{c}^{L} l_{6}(k+1, L-1)\right)\right\} B^{T}(k) \\
& =\Phi d_{2}(k+1, L-1)+\Phi G_{1}(L)\left(H \Phi^{L-k} K_{x}(k, k)\right. \\
& \left.-H d_{2}(k+1, L-1)-d_{4}(k+1, L-1)-d_{6}(k+1, L-1)\right) .
\end{aligned}
$$

The initial value for $d_{1}(k+1, L)$, at $L=k+1$, is $d_{1}(k+1, k+1)$, which is derived as follows:

$$
\begin{aligned}
& d_{2}(k+1, k+1)=\Phi^{k+2} l_{2}(k+1, k+1) B^{T}(k) \\
& =\Phi^{k+2} J_{1}(k+1, k+1) H A(k+1) B^{T}(k) \\
& =\Phi G_{1}(k+1) H \Phi K_{x}(k, k) .
\end{aligned}
$$

Subtracting $l_{3}(k, L-1)$ from $l_{3}(k, L)$, it can be shown that

$$
\begin{aligned}
& l_{3}(k, L)-l_{3}(k, L-1)=\sum_{i=1}^{k}\left(J_{2}(i, L)\right. \\
& \left.-J_{2}(i, L-1)\right) H B(i) \\
& =-J_{2}(L, L)\left(H \Phi^{L} l_{1}(k, L-1)+\Phi_{c}^{L+1} l_{3}(k, L-1)\right. \\
& \left.+\Phi_{c}^{L} l_{5}(k, L-1)\right) .
\end{aligned}
$$

Substituting (A-10) into the equation for $d_{3}(k, L)$, it can be reduced to

$$
\begin{aligned}
& d_{3}(k, L)=\Phi_{c}^{L+2}\left\{l_{3}(k, L-1)-J_{2}(L, L)\left(H \Phi^{L} l_{1}(k, L-1)\right.\right. \\
& \left.\left.+\Phi_{c}^{L+1} l_{3}(k, L-1)+\Phi_{c}^{L} l_{5}(k, L-1)\right)\right\} A^{T}(k) \\
& =\Phi_{c} d_{3}(k, L-1)-\Phi_{c}^{2} G_{2}(L)\left(H d_{1}(k, L-1)\right. \\
& \left.+d_{3}(k, L-1)+d_{5}(k, L-1)\right),
\end{aligned}
$$

where we used the expression for the filter gain $G_{2}(L)=\Phi_{c}^{L} J_{2}(L, L)$ in [13]. The initial value for $d_{3}(k, L)$, at $L=k$, is $d_{3}(k, k)$, which is derived as follows:

$$
\begin{aligned}
& d_{3}(k, k)=\Phi_{c}^{k+2} l_{3}(k, k) A^{T}(k) \\
& =\Phi_{c}^{k+2} \sum_{i=1}^{k} J_{2}(i, k) H B(i) A^{T}(k) \\
& =\Phi_{c}^{k+2} r_{21}(k)\left(\Phi^{T}\right)^{k} \\
& =\Phi_{c}^{2} S_{21}(k) .
\end{aligned}
$$

Here, (A-19) and (A-37), presented in [13], are used.

Subtracting $l_{4}(k+1, L-1)$ from $l_{4}(k+1, L)$, it can be shown that

$$
\begin{aligned}
& l_{4}(k+1, L)-l_{4}(k+1, L-1)=J_{2}(L, L) H A(L) \\
& +\sum_{i=k+1}^{L-1}\left(J_{2}(i, L)-J_{2}(i, L-1)\right) H A(i) \\
& =J_{2}(L, L) H A(L)-J_{2}(L, L)\left(H \Phi^{L} l_{2}(k+1, L-1)\right. \\
& \left.+\Phi_{c}^{L+1} l_{4}(k+1, L-1)+\Phi_{c}^{L} l_{6}(k+1, L-1)\right) .
\end{aligned}
$$

Substituting (A-11) into the equation for $d_{4}(k+1, L)$, it can be reduced to 


$$
\begin{aligned}
& d_{4}(k+1, L)=\Phi_{c}^{L+2}\left\{l_{4}(k+1, L-1)+J_{2}(L, L)(H A(L)\right. \\
& -H \Phi^{L} l_{2}(k+1, L-1)-\Phi_{c}^{L+1} l_{4}(k+1, L-1) \\
& \left.\left.-\Phi_{c}^{L} l_{6}(k+1, L-1)\right)\right\} B^{T}(k) \\
& =\Phi_{c} d_{4}(k+1, L-1)+\Phi_{c}^{2} G_{2}(L)\left(H \Phi^{L-k} K_{x}(k, k)\right. \\
& \left.-H d_{2}(k+1, L-1)-d_{4}(k+1, L-1)-d_{6}(k+1, L-1)\right) .
\end{aligned}
$$

The initial value for $d_{4}(k+1, L)$, at $L=k+1$, is $d_{4}(k+1, k+1)$, which is derived as follows:

$$
\begin{aligned}
& d_{4}(k+1, k+1)=\Phi_{c}^{k+3} l_{4}(k+1, k+1) B^{T}(k) \\
& =\Phi_{c}^{k+3} J_{2}(k+1, k+1) H A(k+1) B^{T}(k) \\
& =\Phi_{c}^{2} G_{2}(k+1) H \Phi K_{x}(k, k) .
\end{aligned}
$$

Subtracting $l_{5}(k, L-1)$ from $l_{5}(k, L)$, it can be shown that

$$
\begin{aligned}
& l_{5}(k, L)-l_{5}(k, L-1)=\sum_{i=1}^{k}\left(J_{3}(i, L)\right. \\
& \left.-J_{3}(i, L-1)\right) H B(i) \\
& =-J_{3}(L, L)\left(H \Phi^{L} l_{1}(k, L-1)+\Phi_{c}^{L+1} l_{3}(k, L-1)\right. \\
& \left.+\Phi_{c}^{L} l_{5}(k, L-1)\right) .
\end{aligned}
$$

Substituting (A-12) into the equation for $d_{5}(k, L)$, it can be reduced to

$$
\begin{aligned}
& d_{5}(k, L)=\Phi_{c}^{L+1}\left\{l_{5}(k, L-1)-J_{3}(L, L)\left(H \Phi^{L} l_{1}(k, L-1)\right.\right. \\
& \left.\left.+\Phi_{c}^{L+1} l_{3}(k, L-1)+\Phi_{c}^{L} l_{5}(k, L-1)\right)\right\} A^{T}(k) \\
& =\Phi_{c} d_{5}(k, L-1)-\Phi_{c} G_{3}(L)\left(H d_{1}(k, L-1)\right. \\
& \left.+d_{3}(k, L-1)+d_{5}(k, L-1)\right) .
\end{aligned}
$$

where we used the expression for the filter gain $G_{3}(L)=\Phi_{c}^{L} J_{3}(L, L)$ in [13]. The initial value for $d_{5}(k, L)$, at $L=k$, is $d_{5}(k, k)$, which is derived as follows:

$$
\begin{aligned}
& d_{5}(k, k)=\Phi_{c}^{k+1} l_{5}(k, k) A^{T}(k) \\
& =\Phi_{c}^{k+1} \sum_{i=1}^{k} J_{3}(i, k) H B(i) A^{T}(k) \\
& =\Phi_{c}^{k+1} r_{31}(k)\left(\Phi^{T}\right)^{k} \\
& =\Phi_{c} S_{31}(k) .
\end{aligned}
$$

Here, (A-24) and (A-37), presented in [13], are used.

Subtracting $l_{6}(k+1, L-1)$ from $l_{6}(k+1, L)$, it can be shown that

$$
\begin{aligned}
& l_{6}(k+1, L)-l_{6}(k+1, L-1)=J_{3}(L, L) H A(L) \\
& +\sum_{i=k+1}^{L-1}\left(J_{3}(i, L)-J_{3}(i, L-1)\right) H A(i) \\
& =J_{3}(L, L) H A(L)-J_{3}(L, L)\left(H \Phi^{L} l_{2}(k+1, L-1)\right. \\
& \left.+\Phi_{c}^{L+1} l_{4}(k+1, L-1)+\Phi_{c}^{L} l_{6}(k+1, L-1)\right) .
\end{aligned}
$$

Substituting (A-13) into the equation for $d_{6}(k+1, L)$, it can be reduced to

$$
\begin{aligned}
& d_{6}(k+1, L)=\Phi_{c}^{L+1}\left\{l_{6}(k+1, L-1)+J_{3}(L, L)(H A(L)\right. \\
& -H \Phi^{L} l_{2}(k+1, L-1)-\Phi_{c}^{L+1} l_{4}(k+1, L-1) \\
& \left.\left.-\Phi_{c}^{L} l_{6}(k+1, L-1)\right)\right\} B^{T}(k) \\
& =\Phi_{c} d_{6}(k+1, L-1)+\Phi_{c} G_{3}(L)\left(H \Phi^{L-k} K_{x}(k, k)\right. \\
& \left.-H d_{2}(k+1, L-1)-d_{4}(k+1, L-1)-d_{6}(k+1, L-1)\right) .
\end{aligned}
$$

The initial value for $d_{6}(k+1, L)$, at $L=k+1$, is $d_{6}(k+1, k+1)$, which is derived as follows:

$$
\begin{aligned}
& d_{6}(k+1, k+1)=\Phi_{c}^{k+2} l_{6}(k+1, k+1) B^{T}(k) \\
& =\Phi_{c}^{k+2} J_{3}(k+1, k+1) H A(k+1) B^{T}(k) \\
& =\Phi_{c} G_{3}(k+1) H \Phi K_{x}(k, k) .
\end{aligned}
$$

(Q.E.D.)

\section{Author's Profile}

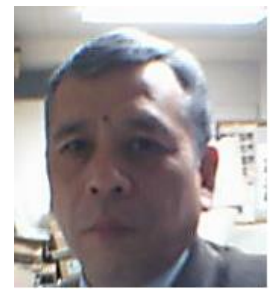

Seiichi Nakamori: Professor, Department of Technology, Faculty of Education, Kagoshima University, mainly interested in stochastic signal estimation and image restoration.

$\mathrm{He}$ received the B.E. degree in Electronic Engineering, Kagoshima University, in 1974 and the Dr. Eng. Degree in Applied Mathematics and Physics from Kyoto University in 1982.

How to cite this paper: Seiichi Nakamori,"RLS Wiener Smoother for Colored Observation Noise with Relation to Innovation Theory in Linear Discrete-Time Stochastic Systems", International Journal of Information Technology and Computer Science(IJITCS), vol.5, no.3, pp.1-12, 2013.DOI: $10.5815 /$ ijitcs.2013.03.01 